\title{
Role of the pion size and flux-tube extension in a baryon-decay model
}

\author{
Fl. Stancu and P. Stassart \\ Institut de Physique B5, Université de Liège, Sart Tilman, B-4000 Liège 1, Belgium
}

(Received 17 June 1988)

\begin{abstract}
A single-flux-tube-breaking mechanism is generalized to three flux tubes and applied to the study of pion decay of nonstrange resonances. The results given by a finite-extension flux tube are compared to those obtained previously from the naive quark-pair-creation model supported by the breaking of an infinite-extension flux tube. The role of the pion wave function and its size are also discussed.
\end{abstract}

The strong-coupling Hamiltonian lattice formulation of QCD has inspired flux-tube models ${ }^{1,2}$ which have successfully been applied to hadron spectroscopy of baryons $^{3-5}$ and mesons. ${ }^{3,6}$ These are usually semirelativistic constituent-quark models, and the interaction between quarks is described by two- and three-body potentials proportional to the string tension of the flux tube.

The strong-coupling limit also provides a theoretical foundation of the meson decay through the breaking of a flux tube. ${ }^{6}$ In the limit where the flux-tube wave-function rms radius becomes infinite ${ }^{6}$ one can recover the so-called naive quark-pair-creation (QPC) model. ${ }^{7,8}$ An alternative theoretical foundation of the QPC model has also been obtained using a strong coupling and hopping-parameter expansion. ${ }^{9}$ In Ref. 9 the expression for the decay width of unstable mesons covers a more general kinematics than that of Ref. 8.

\section{BREAKING OF A FINITE-EXTENSION FLUX TUBE}

A purpose of this paper is to extend the flux-tubebreaking mechanism to the study of baryon decays. The baryon wave function is more complicated than that of the meson and it can be described by two distinct fluxtube configurations. ${ }^{1}$ As shown in Fig. 1 one contains three flux tubes emerging from the three quarks and meeting at $120^{\circ}$ at a common point $r_{4}$. The other appears for interior angles larger than $120^{\circ}$ and contains only two

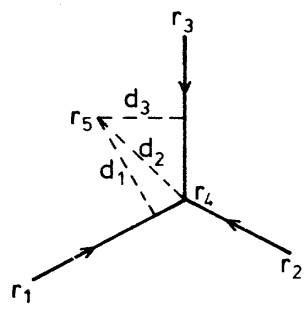

(a)

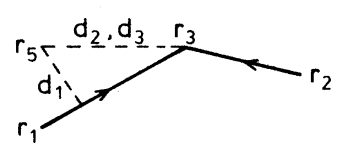

(b)
FIG. 1. Geometry of the decay mechanism by flux-tube breaking at the point $\mathbf{r}_{5}$. flux tubes because the third one collapsed to the common point. Considering an arbitrary point $\mathbf{r}_{5}$ where the pair creation can occur within the volume occupied by the baryon, we define a total flux-tube-breaking amplitude $\gamma\left(\mathbf{r}_{1}, \mathbf{r}_{2}, \mathbf{r}_{3}, \mathbf{r}_{4}, \mathbf{r}_{5}\right)$ as the sum

$$
\gamma\left(\mathbf{r}_{1}, \mathbf{r}_{2}, \mathbf{r}_{3}, \mathbf{r}_{4}, \mathbf{r}_{5}\right)=\sum_{i=1}^{3} \gamma_{i},
$$

where $\gamma_{i}$ is the elementary single flux-tube-breaking amplitude introduced in Ref. 6 for the meson decay. Although both ends of the flux tubes are not source points as in the meson case we still assume for $\gamma_{i}$ the simple form proposed in Ref. 6:

$$
\gamma_{i}=\gamma_{0} \exp \left[-\frac{\sigma^{1 / 2}}{2 \hbar c} d_{i}^{2}\right],
$$

where $\gamma_{0}$ is an adjustable parameter, $\sigma^{1 / 2}=1 \mathrm{GeV} / \mathrm{fm}$ is the string-tension constant, and $d_{i}$ is the shortest distance from $\mathbf{r}_{5}$ to the flux tube $i$. Whenever the perpendicular from $r_{5}$ falls outside the length of the tube we take $d_{i}$ as the distance to the nearest end, i.e., we assume a cigarshaped pair creation region as in Ref. 6. For configurations where one tube collapses, for reasons of continuity we maintain its contribution through the distance from $\mathbf{r}_{5}$ to the collapse point. An example is illustrated in Fig. 1(b). In the case of Fig. 1(a) the common point $r_{4}$ is uniquely defined by $r_{i}(i=1,2,3)$ through the requirement of a minimum confining energy. It is useful to use the Jacobi coordinates

$$
\begin{aligned}
& \boldsymbol{\rho}=\left(\mathbf{r}_{1}-\mathbf{r}_{2}\right) / 2^{1 / 2}, \\
& \lambda=\left(\mathbf{r}_{1}+\mathbf{r}_{2}-2 \mathbf{r}_{3}\right) / 6^{1 / 2}, \\
& \mathbf{R}=\left(\mathbf{r}_{1}+\mathbf{r}_{2}+\mathbf{r}_{3}\right) / 3^{1 / 2},
\end{aligned}
$$

instead of $\mathbf{r}_{i}$. Then $\gamma$ becomes a function of the variables $\rho, \lambda$, and $\mathbf{r}_{5}$.

For the process $R \rightarrow N+M$ where $R, N$, and $M$ are the resonance, the nucleon ground state, and the outgoing meson, respectively, we define the decay transition amplitude as in Ref. 8: 


$$
\langle N M|\hat{T}| R\rangle=\sum_{m}\langle m-m \mid 00\rangle\left\langle\phi_{N} \phi_{M} \mid \phi_{R} \phi_{\mathrm{vac}}^{-m}\right\rangle I_{m}(R ; N, M),
$$

where the $\phi$ 's are the flavor-spin wave functions of $R, N, M$, and of the quark-antiquark pair created from the vacuum in a ${ }^{3} P_{0}$ state and $I_{m}$ is a generalization of the overlap integral of Ref. 10:

$$
\begin{aligned}
I_{m}(R ; N, \boldsymbol{M})=-\left(\frac{3}{4 \pi}\right)^{1 / 2} \frac{2^{3}}{(2 \pi)^{3 / 2}} \delta\left(\mathbf{k}_{M}+\mathbf{k}_{N}\right) \int & d \boldsymbol{\rho} d \lambda d \mathbf{x} d \mathbf{r}_{5} \psi_{R}\left(\boldsymbol{\rho}, \boldsymbol{\lambda}+\left(\frac{8}{3}\right)^{1 / 2} \mathbf{x}\right) \\
& \times \psi_{N}(\boldsymbol{\rho}, \lambda) \exp \left\{i \mathbf{k}_{M} \cdot\left[\left(\frac{2}{3}\right)^{1 / 2} \lambda+\mathbf{x}\right]\right\} \\
& \times \boldsymbol{\epsilon}_{m} \cdot\left(\mathbf{k}_{M}+i \nabla_{\mathbf{x}}\right) \psi_{M}(2 \mathbf{x}) \gamma\left(\boldsymbol{\rho}, \lambda, \mathbf{r}_{5}\right),
\end{aligned}
$$

where $\boldsymbol{\epsilon}_{m}$ is the spherical unit vector:

$$
\boldsymbol{\epsilon}_{ \pm 1}=\mp \frac{1}{2^{1 / 2}}(1, i, 0), \quad \epsilon_{0}=(0,0,1) \text {. }
$$

In Eq. (1.5) $\mathbf{k}_{M}$ and $\mathbf{k}_{N}$ are the momenta of the outgoing meson and nucleon, respectively, and the $\psi$ 's are the spatial parts of the corresponding wave functions, with $x$ the relative internal coordinate of the outgoing pion. The particular case $\gamma=$ const reduces $I_{m}$ to a ninedimensional integral which recovers the naive QPC model of Ref. 8, where $I_{m}$ was expressed in momentum coordinates instead of spatial coordinates as above. The simplification introduced by the QPC model can, therefore, be interpreted as relying on the assumption that the amplitude for the breaking of the tube (string) is equal at all points and independent of the transverse direction from the tube. ${ }^{6}$

An important characteristic ${ }^{11}$ of the QPC model is the nonlocal character of the pion emission operator which is transparent from Eq. (1.5) where $\lambda$ is shifted to $\lambda+\left(\frac{8}{3}\right)^{1 / 2} \mathbf{x}$ in $\psi_{R}$, i.e., proportional to the extension of the pion.

\section{THE ROLE OF THE PION WAVE FUNCTION}

The radial extent and wave function of the pion is an important issue. ${ }^{12}$ In Ref. 13 it has been shown that the decay widths of mesons are quite sensitive to the size of the emitted pion. In Ref. 14 the study of the pion branching ratio in the $N \bar{N}$ annihilation processes favors pion wave functions with rms radii smaller than for other $s$ - and $p$-wave mesons.

In Ref. 10 a finite-size pion with an rms radius of 0.29 fm has been used in the calculations of the decay widths of baryons. The results were globally better than those obtained from a pointlike pion. However some striking discrepancies with respect to experiment remained. The comparison between the finite size and the pointlike results hinted at a pion of a smaller size.

In keeping with the conclusions of Ref. 10 we explore here the effect of using the pion wave function of Ref. 3 which produces a radius of $0.16 \mathrm{fm}$. In principle this would describe the pion better because it includes the one-gluon-exchange contribution through short-range correlations. In this respect it is more consistent with the baryon states ${ }^{4,15}$ as they also include one-gluon exchange.

In addition to one-gluon exchange the three-quark and the quark-antiquark systems are described by the same
QCD-inspired Hamiltonian. ${ }^{1}$ This contains a relativistic kinetic-energy term and an adiabatic potential obtained by minimizing the energy in the gauge fields for fixed quark positions. The baryon states are obtained through the diagonalization of a hyperfine interaction containing spin-spin and tensor parts and their description is extensively given in Refs. 4 and 15 . In that interaction the quark mass $m$ and size $\Lambda$ have been treated as parameters. In the following we shall use baryon states obtained with the so-called "set II" where $m=324 \mathrm{MeV}$ and $\Lambda=0.09 \mathrm{fm}$ (see details in Ref. 15).

The incorporation of the hyperfine interaction into the pion wave function is performed in Ref. 3 through a variational procedure. The values of the hyperfine interaction parameters used there are slightly different than those used for the baryon, i.e., $m=360 \mathrm{MeV}$ and $\Lambda=0.13 \mathrm{fm}$. The strong coupling constant $\alpha_{c}$ and string tension $\sqrt{\sigma}$ are the same in both cases:

$$
\frac{4}{3} \frac{\alpha_{c}}{\hbar c}=0.5, \quad \sigma^{1 / 2}=1 \mathrm{GeV} \mathrm{fm}^{-1} .
$$

The radial part of the pion wave function is given by ${ }^{3}$

$$
\psi_{r}(r)=f^{c}(r)\left[1+u^{\sigma}(r) \sigma_{q} \cdot \sigma_{\bar{q}}\right],
$$

where the central part $f^{c}(r)$ is parametrized as in Ref. 1:

$$
\begin{gathered}
f^{c}(r)=r^{-0.2} \exp \{-0.3965 W(r) \\
\left.-2.1[1-W(r)] r^{1.5}\right\}, \\
W(r)=\frac{1+\exp (-0.15 / 0.05)}{1+\exp [(r-0.15) / 0.05]} .
\end{gathered}
$$
by

The spin-spin correlation part $u^{\sigma}(r)$ is defined in Ref. 3

$$
u^{\sigma}(r)=\beta_{\sigma} \int \frac{e^{-\mu_{\sigma}\left|\mathbf{r}-\mathbf{r}^{\prime}\right|}}{\left|\mathbf{r}-\mathbf{r}^{\prime}\right|} V_{s s}\left(r^{\prime}\right) d^{3} r^{\prime},
$$

with

$$
\beta_{\sigma}=-7 \mathrm{GeV}^{-1} \mathrm{fm}^{-2}, \quad \mu_{\sigma}=10 \mathrm{fm}^{-1},
$$

and

$$
V_{S S}(r)=\frac{4}{3} \frac{\alpha_{s}}{\hbar c} \frac{1}{12 m^{2}} \frac{1}{\Lambda^{3} \pi^{1 / 2}} e^{-(r / 2 \Lambda)^{2}} .
$$

To reduce the volume of numerical computation of the multiple integral (1.5), we parametrized $u^{\sigma}$ by the analytic expression 


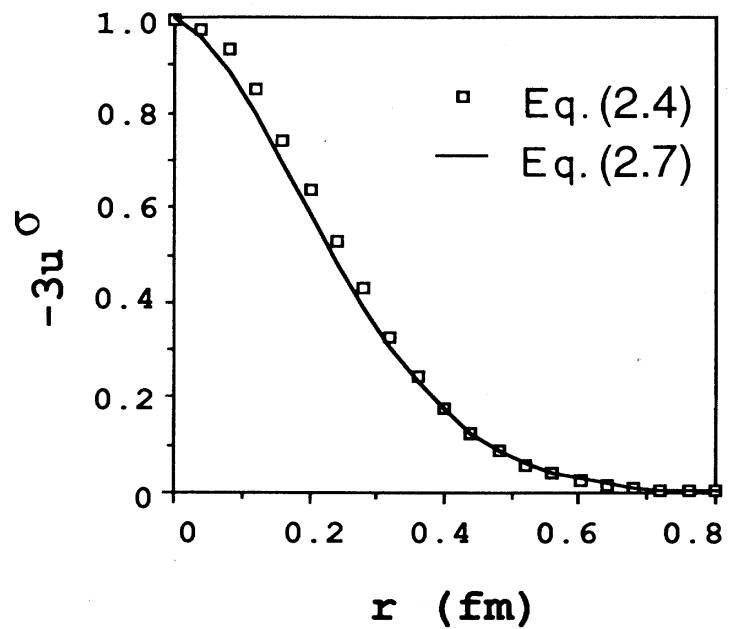

FIG. 2. The numerical solution of the integral (2.4) and its fit by the analytic expression (2.7).

$$
\begin{aligned}
& u^{\sigma}(r)=u^{\sigma}(0) \exp \left\{-\left(\gamma_{1} r+\gamma_{2} r^{2}\right) W(r)\right. \\
& \left.-\gamma_{1.5} r^{1.5}[1-W(r)]\right\}, \\
& u^{\sigma}(0)=-0.332 \text {, } \\
& W=\frac{1+\exp \left(-r_{0} / a\right)}{1+\exp \left[\left(r-r_{0}\right) / a\right]} \text {. }
\end{aligned}
$$

A $\chi^{2}$ fit to the numerical values of (2.4) gave

$$
\begin{aligned}
& \gamma_{1}=0.115 \mathrm{fm}^{-1}, \quad \gamma_{2}=10.5 \mathrm{fm}^{-2}, \\
& \gamma_{1.5}=7.0 \mathrm{fm}^{-3 / 2}, \\
& r_{0}=0.72 \mathrm{fm}, a=0.11 \mathrm{fm} .
\end{aligned}
$$

The numerical evaluation of (2.4) and expression (2.7) are drawn in Fig. 2 as a function of $r$.

\section{DISCUSSION}

To analyze the role of the pion size and of the extension of the flux tube we display in Table I the square root of decay widths calculated in three different ways. Column 1 lists the investigated resonances for which experimental data are available. The main component of each resonance wave function is given in column 2 . In column 3 we reproduce the results of Ref. 10. These have been obtained with $\gamma=$ const and $\psi_{\pi}=f^{c}(r)$ of Eq. (2.3), i.e., without a gluon-exchange contribution in the pion wave function. In column 4 we maintain $\gamma=$ const but use the pion wave function (2.2) with $u^{\sigma}$ from Eqs. (2.7) and (2.8). Results of column 5 correspond to $\gamma$ defined in Eqs. (1.1) and (1.2) and the correlated pion wave function (2.2). In each case $\Gamma^{1 / 2}$ carries the sign of the corresponding transition amplitude defined according to the

\begin{tabular}{|c|c|c|c|c|c|c|}
\hline Resonance & Main component & $\begin{array}{c}\gamma=\text { const } \\
\psi_{\pi}=f_{c}\end{array}$ & $\begin{array}{c}\gamma=\text { const } \\
\psi_{\pi} \text { Eq. }(2.2)\end{array}$ & $\begin{array}{c}\gamma \text { Eqs. (1.1) and (1.2) } \\
\psi_{\pi} \text { Eq. (2.2) }\end{array}$ & Expt. & Status \\
\hline$P_{11}(1440)$ & ${ }^{2} N\left(56^{\prime}, 0^{+}\right) \frac{1}{2}^{+}$ & +20.8 & +12.1 & +16.2 & $10.9_{-3.2}^{+4.8}$ & $* * * *$ \\
\hline$D_{13}(1520)$ & ${ }^{2} N\left(70,1^{-}\right) \frac{3}{2}^{-}$ & +8.4 & +7.1 & +7.5 & $8.3_{-1.2}^{+0.9}$ & $* * * *$ \\
\hline$S_{11}(1535)$ & ${ }^{2} N\left(70,1^{-}\right) \frac{1}{2}^{-}$ & +6.3 & +9.2 & +9.3 & $8.0_{-2.1}^{+3.2}$ & $* * * *$ \\
\hline$S_{11}(1650)$ & ${ }^{4} N\left(70,1^{-}\right) \frac{1}{2}^{-}$ & +2.3 & +7.2 & +7.7 & $9.5_{-2.1}^{+1.9}$ & $* * * *$ \\
\hline$D_{15}(1675)$ & ${ }^{4} N\left(70,1^{-}\right) \frac{5}{2}^{-}$ & +5.6 & +5.1 & +5.4 & $7.6_{-1.1}^{+0.9}$ & $* * * *$ \\
\hline$F_{15}(1680)$ & ${ }^{2} N\left(56,2^{+}\right) \frac{5}{2}^{+}$ & +9.7 & +7.5 & +8.5 & $8.7_{-0.9}^{+0.8}$ & $* * * *$ \\
\hline$D_{13}(1700)$ & ${ }^{4} N\left(70,1^{-}\right) \frac{3}{2}^{-}$ & -4.1 & -3.6 & -3.9 & $3.2_{-1.3}^{+1.0}$ & $* * *$ \\
\hline$P_{11}(1710)$ & ${ }^{2} N\left(70,0^{+}\right) \frac{1}{2}^{+}$ & +1.8 & -3.7 & -3.1 & $4.0_{-1.0}^{+1.1}$ & $* * *$ \\
\hline$P_{13}(1720)$ & ${ }^{2} N\left(56,2^{+}\right) \frac{3}{2}^{+}$ & -7.1 & -11.0 & -11.7 & $5.4_{-1.9}^{+1.7}$ & $* * * *$ \\
\hline$F_{17}(1990)$ & ${ }^{4} N\left(70,2^{+}\right) \frac{7}{2}^{+}$ & -1.8 & -1.1 & -1.2 & $4.2_{-2.1}^{+1.9}$ & $* *$ \\
\hline$F_{15}(2000)$ & ${ }^{4} N\left(70,2^{+}\right) \frac{5}{2}^{+}$ & -2.0 & -1.8 & -1.7 & $1.9_{-0.7}^{+0.7}$ & $* *$ \\
\hline$P_{33}(1232)$ & ${ }^{4} \Delta\left(56,0^{+}\right) \frac{3}{2}^{+}$ & +10.7 & +10.7 & +10.7 & $10.7_{-0.2}^{+0.2}$ & $* * * *$ \\
\hline$P_{33}(1600)$ & ${ }^{4} \Delta\left(56^{\prime}, 0^{+}\right) \frac{3}{2}^{+}$ & -0.2 & -7.4 & -4.1 & $7.0_{-1.8}^{+2.4}$ & $* *$ \\
\hline$S_{31}(1620)$ & ${ }^{2} \Delta\left(70,1^{-}\right) \frac{1}{2}^{-}$ & -0.6 & -2.8 & -2.9 & $6.5_{-1.0}^{+1.0}$ & $* * * *$ \\
\hline$D_{33}(1700)$ & ${ }^{2} \Delta\left(70,1^{-}\right) \frac{3}{2}^{-}$ & -4.8 & -4.3 & -4.5 & $6.1_{-1.7}^{+1.6}$ & $* * * *$ \\
\hline$F_{35}(1905)$ & ${ }^{2} \Delta\left(70,2^{+}\right) \frac{5}{2}^{+}$ & +3.1 & +2.1 & +2.3 & $5.5_{-2.0}^{+2.2}$ & $* * * *$ \\
\hline$P_{31}(1910)$ & ${ }^{2} \Delta\left(70,0^{+}\right) \frac{1}{2}^{+}$ & +0.7 & +5.4 & +6.0 & $6.6_{-1.1}^{+2.5}$ & $* * * *$ \\
\hline$P_{33}(1920)$ & ${ }^{4} \Delta\left(56,2^{+}\right) \frac{3}{2}^{+}$ & +2.0 & +2.4 & +0.5 & $6.6_{-1.3}^{+1.1}$ & $* * *$ \\
\hline$F_{37}(1950)$ & ${ }^{4} \Delta\left(56,2^{+}\right) \frac{7}{2}^{+}$ & -8.7 & -6.6 & -7.2 & $9.8_{-1.4}^{+2.6}$ & $* * * *$ \\
\hline
\end{tabular}
phase conventions of Ref. 16. The last two columns give the square root of the experimental decay width and the

TABLE I. Square root of the decay widths $\Gamma_{N \pi}^{1 / 2}$ in $\mathrm{MeV}^{1 / 2}$. Column 2: main component (Ref. 15). Column 3: the QPC model with a pion of rms radius $0.29 \mathrm{fm}$. Column 4: the QPC model with a pion of rms radius 0.16 fm. Column 5: a finite-extension fluxtube-breaking mechanism and a pion of rms radius $0.16 \mathrm{fm}$. Columns 6 and 7: data and status of resonances (Ref. 17). Each calculated $\Gamma^{1 / 2}$ carries the sign of the associated transition amplitude. The negative-parity states have pure imaginary amplitudes. 
present experimental status ${ }^{17}$ of the resonances.

By comparing columns 3 and 4 one can see that the smaller sized pion described by Eq. (2.2) acts as predicted in Ref. 10. For the resonances $P_{11}(1440), S_{11}(1650)$, $P_{33}(1600), S_{31}(1620)$, and $P_{31}(1910)$ the new pion wave function brings the theoretical values of $\Gamma^{1 / 2}$ much closer to experimental data, lowering the $\chi^{2}$ from 105 to 56 . The calculated sign of the amplitudes associated with the $P_{11}(1440), S_{11}(1650)$, and $S_{31}(1620)$ resonances also correctly reproduces the sign of the helicity amplitude of the radiative decay according to the discussion of Ref. 18 . For the $P_{33}(1600)$ and $P_{31}(1910)$ one cannot make a definite statement about the sign because of experimental uncertainty on the amplitudes $A_{3 / 2}^{P}$ or $A_{1 / 2}^{P}$. The width of the $P_{33}(1920)$ resonance does not change much but it goes in the right direction. For the other resonances the agreement is well maintained except for the $P_{13}(1720)$ resonance where the situation worsens.

At this point it would be useful to be reminded that the pion size is related to the nonlocality of the pion emission operator (1.5) and is important for the decay process. ${ }^{11}$ Such a size should also be consistent with the experimental charge radius ${ }^{19}$

$$
\left\langle r_{\pi}^{2}\right\rangle_{\mathrm{ch}}^{1 / 2}=(0.66 \pm 0.01) \mathrm{fm} \text {. }
$$

It has been shown that the pion radius as seen by the photon is determined almost completely by the intermediate $\rho$ meson, ${ }^{20}$ in agreement with the vectordominance model. ${ }^{21}$ This indicates that the radius of the pion, resulting from the quark confinement as above, must be much smaller than (3.1). Reference 12 gives an upper limit of $0.4 \mathrm{fm}$. Within that picture, the values considered in these calculations, i.e., $0.16 \mathrm{fm}$ and $0.29 \mathrm{fm}$ are both consistent with experiment. .

Columns 4 and 5 indicate that a finite- and an infinite- extension flux tube give results close to each other. A similar conclusion has been drawn in the case of meson decay. ${ }^{6}$ It means that in the integral (1.5) the nonlocality introduced by the naive QPC model plays the dominant role.

The only significant effect of a finite-extension flux tube is felt on the resonances $P_{11}(1440)$ and $P_{33}(1600)$. This can be explained by the fact that they are both mainly radial excitations and have therefore a larger extension. This change goes in the wrong direction in both cases and it affects the $\chi^{2}$ which raises from 56 to 81 . However most of the raise in $\chi^{2}$ comes from the $P_{33}(1920)$ resonance, which should be regarded with care. The value of that width is subject to large statistical error in the Monte Carlo method. Generally, for $\Gamma<1 \mathrm{MeV}$ there is a large ( $>50 \%$ ) numerical error.

With the exception of the $P_{11}(1710)$ the signs of the strong decay amplitudes remain unchanged when passing from column 3 to 4 or 5 . The signs obtained in columns 4 and 5 are the same as those obtained in a harmonicoscillator basis. ${ }^{16}$ For a detailed discussion, see Ref. 18.

In conclusion, it seems that an infinite-extension flux tube, i.e., $\gamma=$ const is a very good approximation in the treatment of the decay widths. Also the present results indicate that the pion wave function of Ref. 3 which contains the one-gluon-exchange effect gives an appropriate description to strong decay of baryons.

\section{ACKNOWLEDGMENTS}

We are grateful to $\mathrm{L}$. Wilets for generous help in the numerical computation and to $J$. Kogut and S. Kumano for kindly providing the variational parameters of Eq. (2.5). The work of one of us (P.S.) was supported by the Institut Interuniversitaire des Sciences Nucléaires, Belgium.
1J. Carlson, J. Kogut, and V. R. Pandharipande, Phys. Rev. D 27, 23 (1983).

${ }^{2}$ N. Isgur and J. Paton, Phys. Rev. D 31, 2910 (1985).

3 J. Carlson, J. Kogut, and V. R. Pandharipande, Phys. Rev. D 28, 2808 (1983).

${ }^{4}$ R. Sartor and Fl. Stancu, Phys. Rev. D 31, 128 (1985).

${ }^{5}$ C. Capstick and N. Isgur, Phys. Rev. D 34, 2809 (1986).

${ }^{6}$ R. Kokoski and N. Isgur, Phys. Rev. D 35, 907 (1987).

${ }^{7}$ L. Micu, Nucl. Phys. B10, 521 (1969); R. Carlitz and M. Kislinger, Phys. Rev. D 2, 336 (1970).

${ }^{8}$ A. Le Yaouanc, L. Oliver, O. Pène, and J.-C. Raynal, Phys. Rev. D 8, 2223 (1973); 9, 1415 (1974); 11, 1272 (1975).

${ }^{9}$ H. G. Dosch and D. Gromes, Phys. Rev. D 33, 1378 (1986).

${ }^{10} \mathrm{Fl}$. Stancu and P. Stassart, Phys. Rev. D 38, 233 (1988).

${ }^{11}$ M. B. Gavela, A. Le Yaouanc, L. Oliver, O. Pène, J.-C. Raynal, and S. Sood, Phys. Rev. D 21, 182 (1980).
${ }^{12}$ V. Bernard, R. Brockmann, and W. Weise, Nucl. Phys. A412, 349 (1984); A440, 605 (1985).

${ }^{13}$ S. Kumano and V. R. Pandharipande, Phys. Rev. D 38, 146 (1988).

${ }^{14}$ A. M. Green and G. Q. Liu, Z. Phys. A (to be published)

${ }^{15}$ R. Sartor and Fl. Stancu, Phys. Rev. D 34, 3405 (1986).

${ }^{16}$ R. Koniuk and N. Isgur, Phys. Rev. D 21, 1868 (1980).

${ }^{17}$ Particle Data Group, M. Aguilar-Benitez et al., Phys. Lett. 170B, 1 (1986).

${ }^{18}$ R. Sartor and Fl. Stancu, Phys. Rev. D 33, 727 (1986).

${ }^{19}$ S. R. Amendolia et al., Nucl. Phys. B277, 168 (1986).

${ }^{20}$ G. E. Brown, M. Rho, and W. Weise, Nucl. Phys. A454, 669 (1986).

${ }^{21}$ J. J. Sakurai, Currents and Mesons (University of Chicago Press, Chicago, 1969). 